\title{
L'analyse des phénomènes physiques, éléments essentiels vers la résolution de problèmes industriels
}

\author{
Jean-Pierre Nadeau ${ }^{\mathrm{a}}$ et Jérôme Pailhes \\ ENSAM, TREFLE UMR CNRS 8508, Esplanade des Arts et Métiers, 33405 Talence, France
}

Reçu le 15 mars 2007, accepté le 7 juin 2007

\begin{abstract}
Résumé - Nous présentons ici les éléments de la prise en compte de la liaison entre les besoins utilisateurs exprimés en terme de critères d'appréciation et les phénomènes physiques mis en jeu lors de l'utilisation du produit. Nous proposons une démarche structurée qui va de l'analyse des moments significatifs jusqu'à l'identification des critères d'appréciation de l'utilisateur et des variables de conception indispensables à la définition du produit. La décomposition de la phase d'utilisation du produit en moments significatifs permet de recenser les actions exécutées par l'utilisateur, d'extraire les effets physiques présents et de les qualifier en terme de pertinence. L'exhaustivité de l'analyse s'appuie sur des outils méthodologiques et permet de définir les grandeurs physiques impliquées associées aux critères et aux variables de conception ainsi que les relations les liant. On peut ainsi impliquer les attentes utilisateur dès la conception préliminaire. Cette démarche est appliquée à l'intégration des besoins « utilisateur » lors de l'ouverture d'un téléphone « foldable ».
\end{abstract}

Mots clés : Attentes utilisateurs / moments significatifs / variables de conception / critères

\begin{abstract}
Physics phenomena analysis, main methodological elements to solve industrial problems. In this paper, we present the different elements of connection between users requirements and physical phenomena created during the use of products. We propose a structured methodology which goes from the analysis of the significant moments until the identification of the appreciation criteria of the user and design variables necessary to the product definition. The time decomposition of the product use in significant moments makes it possible to identify all actions realized by the user, to extract the physical effects and to qualify them in term of relevance. The methodological tools ensure the exhaustiveness of the analysis, to define the physical sizes associated with the criteria and the design variables as well as the relations between the criteria and design variables. Then we can take into account users requirements during preliminary design phase. This methodology is applied to the integration of the user requirements at the time of the opening for a telephone foldable.
\end{abstract}

Key words: Users requirements / significant moments / design variables / criteria

\section{Introduction}

L'objet de la conception des produits et des systèmes est de satisfaire les besoins des utilisateurs. Il est indispensable de mettre en place une méthodologie permettant d'intégrer les sensations utilisateurs en conception. À cette fin, nous proposons une approche méthodologique afin d'assurer l'exhaustivité de l'analyse. Puis, nous présentons les éléments prépondérants de la prise en compte de la liaison entre les besoins perçus et les phénomènes physiques mis en jeu lors de l'utilisation

\footnotetext{
a Auteur correspondant :

jean-pierre.nadeau@bordeaux.ensam.fr
}

du produit. Nous réduirons les grandeurs physiques mises en jeu aux variables de conception et aux critères d'appréciation des utilisateurs pour obtenir un modèle parcimonieux. Le synoptique proposé sur la figure 1 récapitule la totalité de la démarche [1]. Ces attentes peuvent être exprimées sous forme de caractéristiques sensorielles [2] qui seront alors liées aux variables de conception [3] ou, dans le cas de cet article, exprimées sous forme de critères d'appréciation particuliers.

Nous appliquerons cette méthode au cas de la fermeture d'un téléphone « foldable ». La figure 2 définit les composants actifs du téléphone lors de sa fermeture. Comme il sera précisé plus avant, la particularité de ce type de téléphone est, qu'à partir d'un certain angle de 


\section{Nomenclature}

\begin{tabular}{|ll|}
\hline$d$ & Diamètre du fil \\
$D$ & Diamètre moyen \\
$f$ & Coefficient de frottement \\
$f l e ̀ c h e$ & Flèche du ressort \\
$F$ & Force \\
$g$ & Accélération de la pesanteur \\
$G$ & Module d'élasticité transversale \\
$h$ & Profondeur \\
$i$ & Angle d'hélice \\
$I$ & Moment d'inertie quadratique \\
$k$ & Raideur \\
$M$ & Masse \\
$n$ & Nombre de spires utiles \\
$N$ & Effort normal \\
$r$ & Rayon du doigt \\
$R$ & Rayon de la came \\
$\left(R_{\mathrm{e}}\right)_{\mathrm{g}}$ & Limite élastique tangentielle \\
$T$ & Effort tangentiel \\
$x$ & Abscisse \\
$y$ & Nombre de spires d'extrémité \\
& \\
grecques & \\
$\alpha$ & Angle de la came \\
$\theta$ & Position de la came \\
$\psi$ & Angle écran/clavier \\
indices & \\
0 & Enroulement \\
$\mathrm{e}$ & \\
\hline
\end{tabular}

fermeture, il se ferme automatiquement. Le concept utilisé est classique, il s'agit d'un doigt qui pousse sur une came. Ce doigt est mû par un ressort dont la détente est possible lorsque la came liée au clapet du téléphone passe devant lui (la came est un cône de profondeur $h$ et d'angle $\alpha$ ). La première photographie (Fig. 2) montre la came liée complètement au clapet, la deuxième précise le mécanisme de transmission d'effort et la troisième donne l'implantation du guide sur le support. On remarque aussi sur cette troisième photographie la came implantée dans le clapet (découpé partiellement). Le schéma en coupe permet de comprendre que la translation du doigt génère la rotation de la came et du clapet.

Des tests auprès d'utilisateurs de téléphone ont permis de définir des critères d'appréciation [4]. Une comparaison des différents téléphones est alors possible. Les utilisateurs définissent leurs vœux concernant l'effort et l'angle de fermeture ainsi que la vitesse de rotation de l'écran. Le tableau 1 précise les critères d'appréciation $(\mathrm{Cr})$ intervenant lors de la fermeture automatique : angle et vitesse de rotation de l'unité d'écran. L'effort de fermeture ou d'ouverture (récepteurs somesthésiques) n'est pas pris en compte car il n'intervient pas lorsque le téléphone se ferme automatiquement. Cette phase de recherche des critères

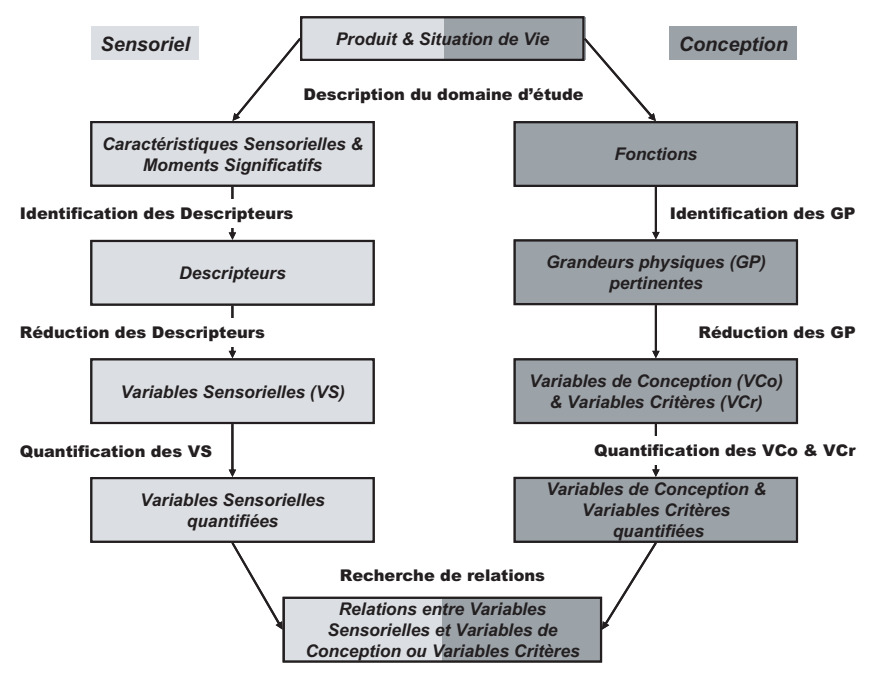

Fig. 1. Synoptique de la démarche parallèle.

Tableau 1. Critères d'appréciation de l'utilisateur.

\begin{tabular}{lccc}
\hline Critère d'appréciation $C r$ & Ouverture & Fermeture \\
\hline Angle position écran/support $\psi$ & $\psi_{\mathrm{o}}$ & $\psi_{\mathrm{f}}$ \\
\hline Vitesse de rotation à un angle donné $\theta$ & $\mathrm{d} \theta_{\mathrm{o}} / \mathrm{d} t$ & $\mathrm{~d} \theta_{\mathrm{f}} / \mathrm{d} t$ \\
\hline
\end{tabular}

liés aux attentes utilisateurs peut s'avérer délicate, des éléments d'aide sont fournis dans [3].

\section{Moments significatifs et situations de vie}

Tout au long de son cycle de vie, un produit rencontre des situations de vie particulières. Le concepteur énumère et analyse ces phases qui permettent de définir les fonctions de service que devra assurer le produit et les contraintes qu'il devra subir (fonctions de premier niveau). En particulier, la fermeture et l'ouverture sont des situations de vie à prendre en compte lors de la conception du téléphone « foldable».

La notion de situation de vie a un défaut, elle ne peut, à cause de la faiblesse de la sémantique liée à sa description, que décrire une phase statique. Cela ne suffit pas car, le plus souvent, le problème, fruit de successions d'événements élémentaires, est dynamique. À l'instar de l'analyse sensorielle [5], nous nommons ces évènements : moments significatifs [6]. Le GRAFCET est un outil facile d'utilisation pour représenter la séquencialité de ces moments (Fig. 3). Il permet, à la fois, d'exprimer la succession des moments et de définir les paramètres et récepteurs sensoriels qui conditionnent les passages de l'un à l'autre.

Dans la situation de vie «fermeture », l'unité écran manœuvrée par la main de l'utilisateur (déblocage et conduite) permet au ressort de se détendre (échappement) et de déplacer le doigt qui assure alors la rotation du clapet (donc de l'unité d'écran) via la came. À chaque étape, le contrôle et la transition sont assurés par l'utilisateur qui récupère des sensations somesthésiques, visuelles et auditives. 


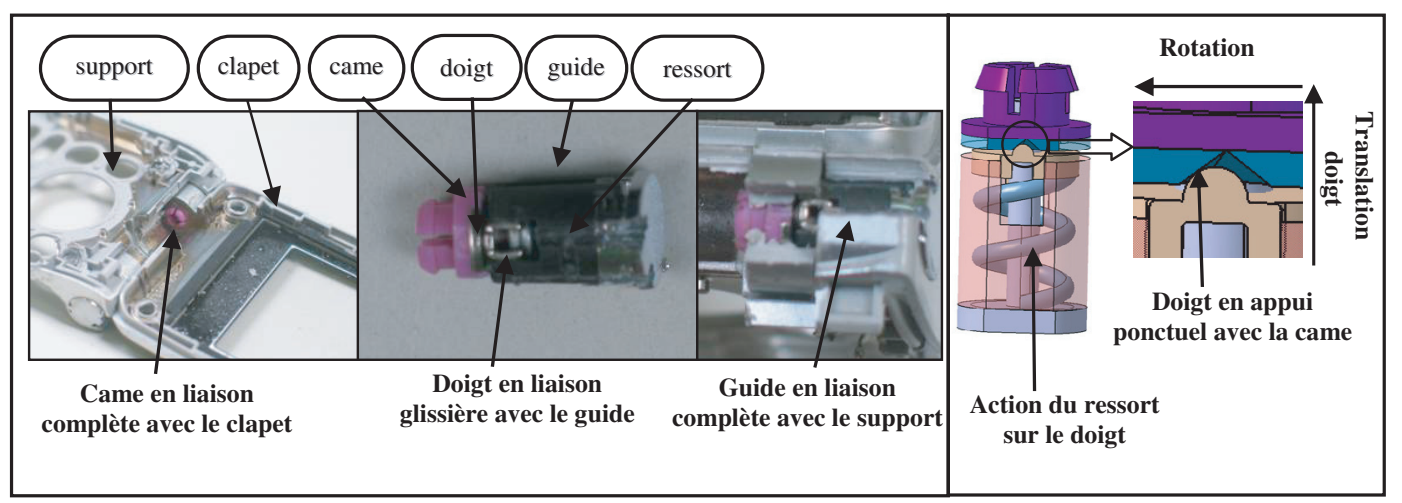

Fig. 2. Composants impliqués dans la fermeture du téléphone.
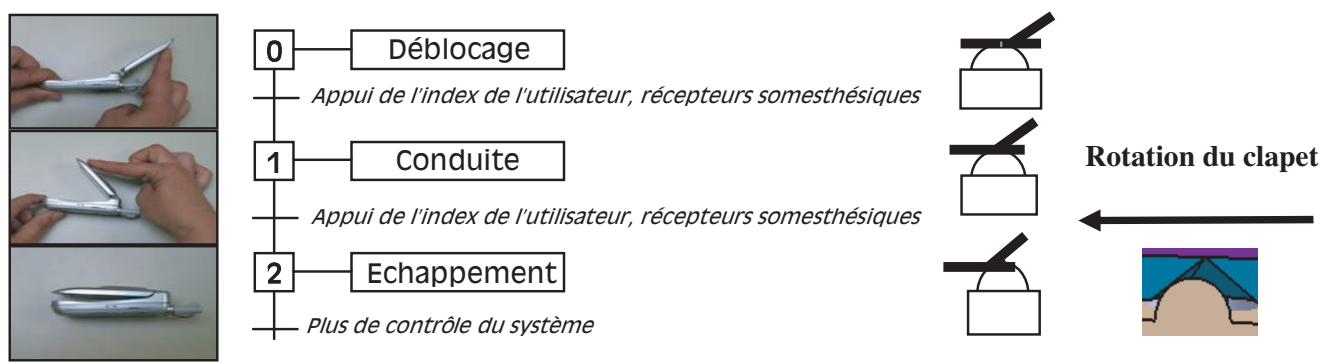

Fig. 3. GRAFCET des moments significatifs pour la fonction « fermer le téléphone ».

Les récepteurs mis en jeu lors des transitions des deux premiers moments significatifs (déblocage et conduite) sont des récepteurs somesthésiques. La somesthésie est associée au retour d'effort, c'est-à-dire ici aux actions entre l'unité écran et l'index de l'utilisateur. La somesthésie est la sensibilité aux diverses excitations subies par le corps, à l'exception de celles provenant des organes sensoriels. Elle comprend les sensations extéroceptives (tact, pression, chaud, froid), les sensations proprioceptives (musculaires et tendineuses) et les sensations douloureuses. Ces sensations récupérées, liées à des habitudes ou dans la plupart des cas à un apprentissage, permettent un contrôle de l'action désirée. A contrario, pour le moment significatif « échappement », il n'y plus de contact entre l'index de l'utilisateur et l'unité d'écran et aucun récepteur sensoriel vient en relève. La vue et l'ouïe sont bien excitées mais ne permettent pas d'action corrective de contrôle (rapidité de l'échappement et bruit présent qu'en fin de fermeture).

\section{Phénomènes physiques mis en jeu et grandeurs physiques pertinentes}

\subsection{Rôle et redondance des outils méthodologiques utilisés}

Afin de définir les grandeurs physiques que l'on peut associer aux critères émis par les utilisateurs, il faut identifier les flux fonctionnels utiles et induits par la satisfaction des fonctions de premier niveau. À cette fin, plusieurs outils sont à notre disposition et la redondance d'une partie de ces outils assure l'exhaustivité de l'analyse. Les outils que nous préconisons regroupent :

- l'organigramme technique du produit étendu aux milieux extérieurs (Fig. 4) [7]. Il permet d'énumérer les composants et les milieux extérieurs,

- la première loi d'évolution des systèmes techniques (Fig. 5) [8]. Elle identifie les composants et les flux fonctionnels concernés,

- le bloc diagramme fonctionnel impliqué dans l'analyse (Fig. 6) [9]. Il représente les flux utiles et induits,

- des tableaux d'analyse physique [10]. Ils aident à l'expression des flux utiles et induits,

- les lois physiques,

- les lois de conservation (masse, énergie, quantité de mouvement, moment cinétique),

- les lois de comportement liées aux composants.

Ces outils méthodologiques permettent un gain de temps au stade de la conception préliminaire et diminuent les risques d'erreurs.

\subsection{Organigramme technique étendu}

Un système fait intervenir plusieurs composants regroupés dans l'organigramme technique étendu aux milieux extérieurs. L'unité clavier est tenue par la main de l'utilisateur et l'écran est fermé par l'index de l'autre main, ils constituent les milieux extérieurs pertinents de la situation de vie «fermeture ». Au premier niveau, le téléphone est constitué par 2 unités : l'unité clavier et l'unité d'écran. À chaque niveau d'un organigramme 


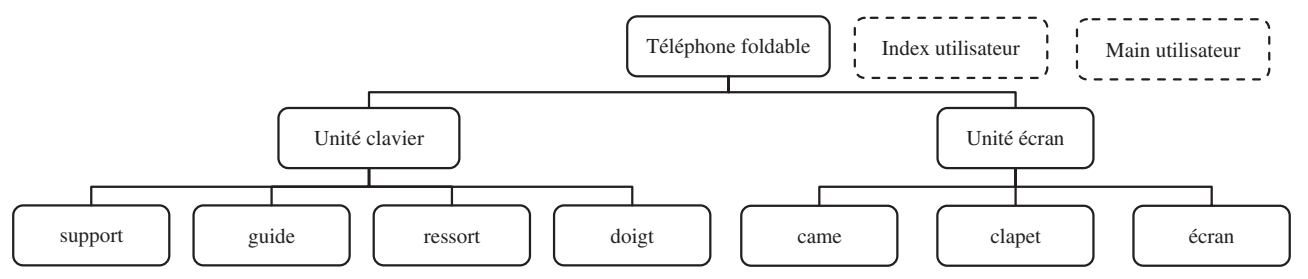

Fig. 4. Organigramme technique étendu du téléphone foldable.

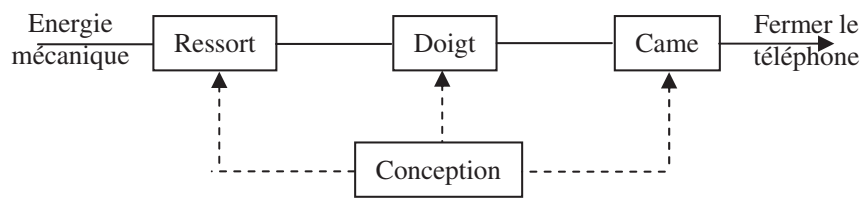

Fig. 5. Loi d'intégralité des parties pour la fonction « fermer le téléphone ».

technique, les composants sont liés par des composants d'interaction. Dans l'évolution des systèmes, ces composants sont souvent standard puis tendent à s'éliminer, la fonction d'interaction s'intègre alors dans un des composants. C'est le cas du téléphone où deux unités sont en liaison pivot, cette liaison est assurée par le guide, partie intégrante de l'unité clavier. Pour préciser le fonctionnement du téléphone nous exprimons l'organigramme technique jusqu'au niveau 2 (Fig. 4).

\subsection{Première loi d'évolution des systèmes techniques}

La réalisation d'une fonction implique la transformation d'une énergie. La première loi d'évolution des systèmes techniques issue de la théorie TRIZ [8] permet de définir plusieurs éléments : une énergie initiale, un moteur qui la transforme, un transmetteur qui transmet l'énergie transformée et un opérateur qui réalise l'action. Chaque élément comporte un ou plusieurs composants de l'organigramme technique. Le flux fonctionnel d'énergie passe dans les composants et à leurs interfaces (composants d'interaction). La figure 5 met en évidence le moteur (ressort), le transmetteur (doigt) et l'opérateur (came) concernant la satisfaction de la fonction de service «fermer le téléphone » lors du moment significatif «échappement ». Le contrôle, dans la phase « échappement », ne se fait pas par l'utilisateur mais est dû au concepteur du système qui doit maîtriser l'angle d'échappement par des choix de variables de conception.

Les différents mouvements sont le fruit du flux fonctionnel d'énergie mécanique, la conservation de la quantité de mouvement de chaque composant va permettre d'exprimer le critère lié au mouvement soit la vitesse de rotation.

\subsection{Bloc diagramme fonctionnel}

Le bloc diagramme fonctionnel permet de préciser l'évolution des flux fonctionnels à travers tous les

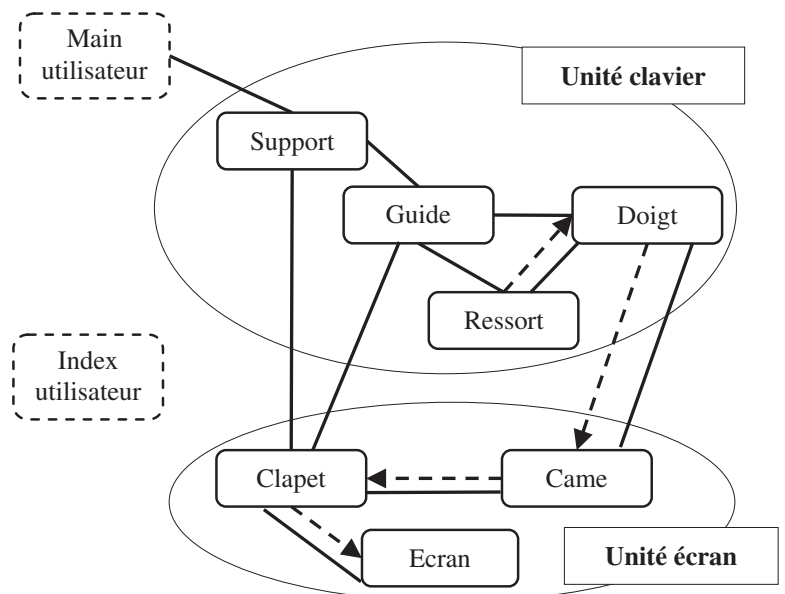

Fig. 6. Bloc diagramme fonctionnel pour la fonction « fermer le téléphone » durant le moment significatif « échappement ».

composants en contact (trait fin) au niveau considéré et de définir des effets induits. La figure 6 précise le cheminement du flux d'énergie mécanique (trait pointillé) entre certains composants pour la fonction étudiée. Nous avons dans notre cas des composants en contact réel (doigt/came, doigt/guide), donc des effets induits dus au frottement. Le tableau 2 récapitule les effets produits lors d'un contact réel.

\subsection{Lois physiques}

\subsubsection{Variables de conception et critères}

La figure 7 présente les variables de conception de cette partie du téléphone. Les variables de conception (VCo) définissent le produit au stade de la conception préliminaire, valuées, elles permettent de différencier 2 solutions.

Il s'agit maintenant de mettre en place les lois physiques qui permettent d'exprimer les critères d'appréciation $(\mathrm{Cr})$ en fonction des variables de conception et de grandeurs physiques pertinentes du problème.

Le premier critère issu des attentes utilisateurs, l'angle de fermeture, est directement lié aux variables de conception $(h, \alpha, r, R)$ et s'exprime simplement par une équation de fermeture géométrique soit :

Angle unité d'écran/unité support :

$$
\psi_{\mathrm{f}} \cong \frac{h \sin \alpha-r \cos \alpha}{R}
$$


Tableau 2. Tableau d'analyse des flux, contact solide/solide.

\begin{tabular}{llll}
\hline $\begin{array}{l}\text { Surfaces en } \\
\text { contact }\end{array}$ & Effet produit & Caractérisation & Nom de la loi \\
\hline $\begin{array}{l}\text { Zone de contact } \\
\text { localisée }\end{array}$ & Champ de pression & Vitesse relative nulle : adhérence & $\begin{array}{l}\text { Hertz (action } \\
\text { normale) } \\
\text { Coulomb }\end{array}$ \\
& Flux d'énergie thermique & $\begin{array}{l}\text { Vitesse relative non nulle : frottement } \\
\text { Vitesse relative non nulle : frottement }\end{array}$ & \\
& Flux d'énergie sonore & Vitesse relative non nulle : frottement & Énergie de \\
& $\begin{array}{l}\text { Flux d'énergie de } \\
\text { déformation }\end{array}$ & Déformation du solide & $\begin{array}{l}\text { Matage (action } \\
\text { normale) }\end{array}$ \\
\hline $\begin{array}{l}\text { Zone de contact } \\
\text { surfacique }\end{array}$ & Champ de pression & Vitesse relative nulle : adhérence & Coulomb \\
\hline & & Vitesse relative non nulle : frottement & \\
\hline & $\begin{array}{l}\text { Flux d'énergie thermique } \\
\text { Flux d'énergie sonore }\end{array}$ & Vitesse relative non nulle : frottement & Vitesse relative non nulle : frottement \\
& $\begin{array}{l}\text { Flux d'énergie de } \\
\text { déformation }\end{array}$ & Déformation du solide & $\begin{array}{l}\text { Énergie de } \\
\text { déformation }\end{array}$ \\
\hline
\end{tabular}

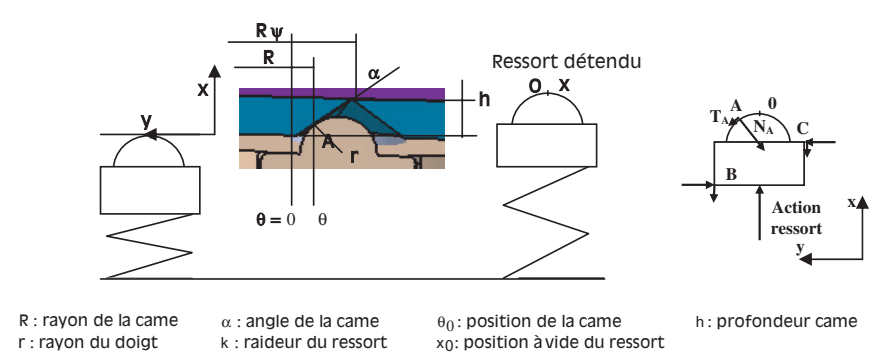

Fig. 7. Évolution du doigt durant la fermeture et actions de contact.

Le second critère (vitesse de rotation de l'unité écran), critère dynamique, impose d'exprimer les lois de conservation de la quantité de mouvement pour chaque entité (moteur, transmetteur, opérateur).

\subsubsection{Conservation de la quantité de mouvement du moteur : équilibre du ressort (somme des forces en projection sur l'axe $x$ )}

Exprimer l'équilibre du ressort impose de descendre à un niveau systémique inférieur. En effet, la définition du ressort en terme de conception, impose de découper le ressort en plusieurs parties, les deux spires particulières d'extrémité et les $n$ spires intermédiaires. Nous donnons en annexe le modèle global de conception et de dimensionnement d'un ressort de compression. Le traitement de ce modèle permet d'exprimer la raideur $k$, grandeur physique caractéristique du ressort au niveau 2 de l'organigramme technique (Fig. 4). Ainsi on peut exprimer l'action d'interaction entre le ressort et le doigt, qui peut s'exprimer sous la forme d'une loi de comportement [11] :

$$
F_{\text {ressort }}=k \cdot \text { flèche }
$$

La flèche est une grandeur physique pertinente de l'état $\mathrm{du}$ ressort selon le moment significatif.

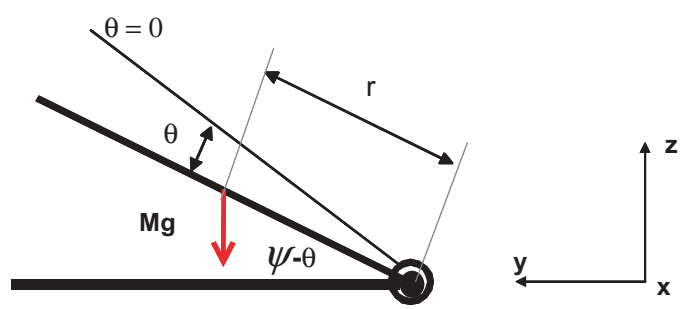

Fig. 8. Unité écran.

3.5.3 Conservation de la quantité de mouvement du transmetteur : équilibre du doigt (somme des forces en projection sur l'axe $x$ )

On l'a vu précédemment, le contact entre la came et le doigt est un contact réel. La loi de comportement des contacts réels, pour une hypothèse de contact ponctuel et de glissement, s'exprime par l'équation de liaison entre l'effort normal et l'effort tangentiel (loi de Coulomb), soit :

$$
\left|T_{\mathrm{A}}\right|=\left|N_{\mathrm{A}}\right| \cdot f
$$

L'équilibre du doigt s'écrit :

$$
k \cdot \text { flèche }-2\left|N_{\mathrm{A}}\right| \sin \alpha-2\left|T_{\mathrm{A}}\right| \cos \alpha-\left|T_{\mathrm{B}}\right|-\left|T_{\mathrm{C}}\right|=0
$$

\subsubsection{Conservation de la quantité de mouvement de l'opérateur : équilibre de l'unité d'écran (somme des moments en projection sur l'axe $x$ )}

La came (opérateur) est liée complètement au clapet et à l'écran, on doit donc traduire l'équilibre de l'unité d'écran. Les grandeurs physiques liées à l'unité d'écran sont sa masse, la position de son centre de gravité et son moment d'inertie quadratique par rapport à l'axe d'interaction entre les deux unités.

$2\left|N_{\mathrm{A}}\right| R \cos \alpha-2\left|T_{\mathrm{A}}\right| R \sin \alpha \pm M g r \cos (\psi-\theta)=I \frac{\mathrm{d}^{2} \theta}{\mathrm{d} t^{2}}$ 
Tableau 3. Grandeurs physiques pertinentes.

\begin{tabular}{ll}
\hline Grandeurs physiques & Notations \\
\hline Flèche du ressort & flèche \\
Raideur du ressort & $k$ \\
Moment d'inertie de l'unité d'écran & $I$ \\
\hline
\end{tabular}

En conception préliminaire, on peut négliger le frottement. Les effets de la gravité ne sont pas du même ordre que les effets du ressort. Ces hypothèses réduisent l'expression (5) à :

$$
2\left|N_{\mathrm{A}}\right| R \cos \alpha=I \frac{\mathrm{d}^{2} \theta}{\mathrm{d} t^{2}}
$$

Le tableau 3 récapitule les grandeurs physiques pertinentes du moment significatif « échappement » dans la situation de vie « fermeture .

\section{Réduction des grandeurs physiques pertinentes aux variables de conception VCo et aux critères $\mathrm{Cr}$}

Nous cherchons à réduire le nombre de grandeurs physiques, pour cela nous allons les exprimer en fonction des variables de conception et des critères d'appréciation. Si une grandeur physique s'exprime en fonction de critères et de variables de conception, c'est une variable auxiliaire et elle n'est pas pertinente.

La flèche est dans ce cas. En effet, ce sont le doigt et la came qui contraignent le ressort. Par une équation de fermeture géométrique issue d'une fonction contrainte liée au fonctionnement du téléphone (angle de fermeture imposé), on peut exprimer la flèche en fonction des variables de conception du doigt et de la came. $x_{0}$, abscisse initiale du sommet du doigt, constitue la référence.

$$
\text { flèche }=x_{0}-\frac{R \theta}{\operatorname{tg} \alpha}+r \sin \alpha-r
$$

On l'a vu, la raideur $k$ pourrait s'exprimer en fonction des variables de conception du ressort (diamètre du fil, nombre de spires, diamètre d'enroulement, module d'élasticité transversale, limite élastique, hauteur à vide) mais nous ne sommes pas à ce niveau systémique. À notre niveau, le ressort n'est connu que par sa raideur, grandeur physique à conserver.

Le moment quadratique $I$ de l'unité écran est aussi lié à la définition de l'écran, il s'exprime exclusivement en fonction des variables de conception de l'écran. À notre niveau, l'unité écran n'est connue que par son moment quadratique $I$, grandeur physique à conserver.

En regroupant les équations (1), (4)-(7) et par intégration, la solution analytique est alors évidente :

$$
\begin{aligned}
\frac{\mathrm{d} \theta_{\mathrm{f}}}{\mathrm{d} t} & =\frac{B}{A} \sin \left[\operatorname{Arccos}\left(1-\frac{A \psi_{\mathrm{f}}}{B}\right)\right] \text { avec } \\
A & =\frac{k R}{I(\operatorname{tg} \alpha)^{2}} \text { et } B=\frac{k}{I \operatorname{tg} \alpha}\left(x_{0}+r \sin \alpha-r\right)
\end{aligned}
$$

Ainsi, nous avons exprimé les deux critères $\psi_{\mathrm{f}}$ et $\mathrm{d} \theta_{\mathrm{f}} / \mathrm{d} t$ (Éqs. (1) et (8)) en fonction, exclusivement, des 4 variables de conception au niveau 2 soit $R, \alpha, r$ et $h$ et des 2 grandeurs physiques $k$ et $I$. Les attentes utilisateurs peuvent ainsi rapidement donner lieu à la définition de téléphones différents en conception préliminaire. On peut remarquer que le système ainsi obtenu est à 6 degrés de liberté. La hiérarchisation des choix se fera à l'aide d'autres contraintes.

\section{Conclusion}

Il est indispensable d'intégrer les attentes utilisateurs dès la conception préliminaire. À cette fin, nous devons les exprimer sous forme de critères à intégrer dans les cahiers des charges.

Nous avons présenté une démarche structurée pour lier ces critères aux variables de conception et ainsi faciliter leur prise en compte. Cette démarche s'appuie sur des outils d'analyse (organigramme technique étendu aux milieux extérieurs, première loi d'analyse des systèmes techniques, bloc diagramme fonctionnel, tableau d'analyse des phénomènes physiques) afin de réduire le temps d'étude et de fiabiliser l'analyse. Enfin, l'expression des critères et des lois physiques concernées en fonction des grandeurs physiques pertinentes et des variables de conception nous donne les relations qui décrivent la situation réelle. Puis, on réduit chaque grandeur physique en l'exprimant, si possible, en fonction des variables de conception.

Les relations obtenues sont utilisées pour la définition du système en adéquation avec les attentes utilisateurs. Ainsi, une fois les attentes utilisateurs identifiées proprement, il est alors possible de les traiter de la même manière que toute autre contrainte du cahier des charges.

\section{Annexe : Expression de la raideur d'un ressort de compression hélicoïdal}

Les variables de conception du ressort sont le diamètre du fil, le nombre de spires, le diamètre d'enroulement, le module d'élasticité transversale, la limite élastique du matériau et la hauteur à vide.

La conception d'un ressort de compression est basée sur deux hypothèses :

- les extrémités se réduisent à y spires non actives,

- les $n$ spires actives sont sollicitées en torsion pure.

L'hypothèse de torsion pure conduit aux relations classiques suivantes :

Tenue mécanique du ressort, limitation des contraintes tangentielles en dessous de la limite élastique du matériau $\left(R_{\mathrm{e}}\right)_{\mathrm{g}}$ :

$$
\tau_{\max i}=\frac{8 F D_{\mathrm{e}}}{\pi d^{3}} w \leqslant\left(R_{\mathrm{e}}\right)_{\mathrm{g}}
$$


Le coefficient de pondération $w$ dit facteur de Wahl [12] vient corriger l'écart à l'hypothèse de torsion pure et rajoute les effets de cisaillement. Il dépend de la géométrie du ressort (rapport $C=D_{\mathrm{e}} / d$ ), soit :

$$
w=\frac{4 C-1}{4 C-4}+\frac{0,615}{C}
$$

\section{Déformation du ressort, expression de la raideur :}

flèche $=\frac{8 D_{\mathrm{e}}^{3} n}{G d^{4}} F$ d'où l'expression de la raideur :

$$
k=\frac{G d^{4}}{8 D_{\mathrm{e}}^{3} n}
$$

Mais pour définir réellement le ressort, il faut aussi vérifier des contraintes de conception :

\section{Contraintes de limitation du flambage:}

$$
\frac{\text { flèche }}{x_{0}} \leqslant-0,104 \frac{h_{0}}{D_{\mathrm{e}}}+1,04
$$

\section{Contraintes de fabrication :}

$$
4<\frac{D_{\mathrm{e}}}{d}<12 \text { et } n+y=\text { entier }+\frac{1}{2}
$$

\section{Contrainte de montage :}

$$
D_{\mathrm{e}}+d+\Delta D_{\mathrm{e}}<D \text { alésage }
$$

Contrainte de validité de l'hypothèse générale de torsion pure :

$$
\operatorname{tg} i \leqslant 0,12
$$

\section{Contrainte de standardisation :}

$$
D_{\mathrm{e}}=\text { variable entière énumérée }
$$

Catalogue de produits semi-finis : possibilité de stock de fil :

Les fils d'acier pour réaliser des ressorts sont disponibles pour différents diamètres $(d)$ et différents matériaux $\left(\left(R_{\mathrm{e}}\right)_{\mathrm{g}}\right)$. Les variables $d$ et $\left(R_{\mathrm{e}}\right)_{\mathrm{g}}$ sont donc des variables énumérées.

La résolution du problème de conception d'un ressort conduit à plusieurs ressorts compatibles avec les contraintes.

\section{Références}

[1] R. Doré, Intégration des sensations utilisateurs en conception préliminaire, application au ski parabolique et au virage de base, thèse de doctorat, ENSAM, 2004

[2] F. Depledt, Évaluation sensorielle, Manuel méthodologique, collection sciences et techniques agroalimentaires, Tech. \& Doc., Lavoisier, Paris, 1998

[3] R. Doré, J. Pailhès, X. Fischer, J.-P. Nadeau, Identification of sensory variables towards the integration of user requirements into preliminary design, Inter. J. Industrial Ergonomics 37 (2007) 1-11

[4] Z. Zalila, A. Guénand, J. Martin-Lopez, Application of expertons theory in the sensory analysis of cell phone flaps, Quality Engineering 17 (2005) 727-734

[5] M. Meilgard, G.V. Civile, B.T. Carr, Sensory evaluation techniques, CRC Press, 1999

[6] R. Doré, J. Pailhès, X. Fischer, J.-P. Nadeau, Integration of user's requirements in preliminary design: application to parabolic ski in basic turn, PLM05, Inter. Conf. on product lifecycle management, Lyon, 2005

[7] J. Pailhès, J.-P. Nadeau, D. Scaravetti, Intégration des flux fonctionnels pour l'aide à la décision en conception préliminaire, $17^{\mathrm{e}}$ Congrès Français de Mécanique, Troyes, 2005

[8] S.D. Savransky, Engineering of creativity, Introduction to TRIZ methodology of inventive problem solving, Florida, Boca Raton, CRC Press, 2000

[9] D. Scaravetti, J.-P. Nadeau, J. Pailhès, P. Sébastian, Structuring of embodiment design problem based on the product lifecycle, Inter. J. Product Lifecycle Management 2 (2005) 47-70

[10] J.-P. Nadeau, J. Pailhès, Intégration de l'innovation et des sensations utilisateur en conception préliminaire par le biais de l'analyse fonctionnelle, Ingénierie de la conception et cycle de vie du produit, Chapitre 2, 43-62, Traité IC2, ISBN : 2-7462-1214-5, Hermès, Paris, 2006

[11] M.F. Ashby, Materials selection in mechanical design, Butterworth-Heinemann, Read Educational \& Professional Ldt, 1999

[12] A.M. Wahl, Mechanical springs, Penton Publishing Company, 1944 